\title{
MODELO DE PONTO CRÍTICO PARA ESTIMAR DANOS CAUSADOS PELA MELA NA CULTURA DO FEIJOEIRO
}

\author{
${ }^{\bullet}$ Felipe Rafael Garcés Fiallos \\ Unidad de Investigación Cientifica y Tecnológica, Universidad Técnica Estatal de Quevedo, km 7 vía \\ Quevedo - El Empalme, C. P. 73. Mocache, Los Ríos, Ecuador. ${ }^{\circledR}$ felipegarces23@yahoo.com
}

Resumo

$\mathrm{N}_{\mathrm{c}}^{\mathrm{s}}$ o Equador, o feijão (Phaseolus vulgaris L.) é consumido quase pela totalidade da população, sendo também fonte de renda para pequenos e médios produtores. Uma das doenças mais importantes é a mela, ou podridão radicular de rizoctonia, causada pelo fungo necrotrófico Rhizoctonia solani Khun (teleomorfo Thanatephorus cucumeris Frank). Objetivou-se determinar a redução no rendimento de grãos causados pela infecção natural da mela, em diferentes cultivares de feijão, na safra agrícola 2010, no município de Quevedo, Equador. O delineamento experimental foi em blocos casualizados, com sete tratamentos e quatro repetições. $\mathrm{O}$ gradiente da intensidade da doença foi gerado pela variabilidade genética entre cultivares de feijoeiro. A avaliação de severidade (\% de área foliar necrosada) da doença foi determinada no estádio fenológico R7 (desenvolvimento de legume). A colheita foi realizada em aos 124 dias pós-semeadura, para quantificação do rendimento. A equação da função de dano obtida foi $\mathrm{R}=$ 4,257.50 - 33.16 S, onde R é o rendimento de grãos e $\mathrm{S}$ a severidade $(\%)$ da doença, com $\mathrm{R}^{2}=0.82$ e $\mathrm{p}<0.0001$. A equação resultante foi normalizada para $1,000 \mathrm{~kg}$, sendo esta $\mathrm{R}=1,000.0-7.79 \mathrm{~S}$. Concluiu-se que a equação gerada da função de dano pode ser utilizada no cálculo do limiar de dano econômico (LDE), uma alternativa racional indicadora do momento para a aplicação de controle químico da mela em cultivares suscetíveis, mantendo a rentabilidade do agricultor e ser amigável com o meio ambiente.

Palabras claves: Phaseolus vulgaris, podridão radicular de rizoctonia, Rhizoctonia solani.

\section{Critical-point yield model to appraise damage caused by leaf blight in bean crop}

Tn Ecuador, the bean (Phaseolus vulgaris L.) is consumed by the population majority, and also a source of income for small and medium producers. One of the most important disease is the blight or root rot rhizoctonia, caused by the necrotrophic fungus Rhizoctonia solani Kuhn (teleomorph Thanatephorus cucumeris Frank). The objective was to determine the reduction in grain yield caused by natural infection of leaf blight disease in different cultivars of beans, the crop year 2010, the city of Quevedo, Ecuador. The experimental design was randomized blocks with seven treatments and four replicates, which were used to generate gradients of disease, while having the genetic variability among the materials. The assessment of severity (\% leaf area necrotic.plot $\left.{ }^{-1}\right)$ of the disease was in the R7 growth stage (pod development). The crop was harvested at the 124 days post-seeding, to quantify the yield. The equation of the damage function obtained was $\mathrm{R}=4,257.50$ to $33.16 \mathrm{~S}$, where $\mathrm{R}$ is yield and $\mathrm{S}$ is the severity $(\%)$ of the disease, with $\mathrm{R}^{2}=0.82$ and $\mathrm{P}<0.0001$. With the resulting equation was normalizes to $1,000 \mathrm{~kg}$, which is $\mathrm{R}=1,000.0$ to $7.79 \mathrm{~S}$. It was concluded that the generated damage equation can be used to calculate the economic damage threshold (EDT), which is an alternative indicator of the time for application of chemical control of blight on susceptible cultivars, while maintaining the profitability of the farmer and be friendly with the environment.

Key words: Phaseolus vulgaris, blight or root rot for rhizoctonia, , Rhizoctonia solani

\section{INTRODUÇÃO}

$\mathrm{O}$ feijão (Phaseolus vulgaris L.) é a dieta básica dos países em desenvolvimento, por seu preço accessível e também por ser fonte de proteínas vegetais e ferro. Araya \& Hernández (2006) menciona que esta

Recibido: 29-Noviembre-2010. Recibido en forma corregida: 25-Marzo2011. Aceptado: 24-Junio-2011.

Publicado como NOTA TÉCNICA en Ciencia y Tecnología 4(1): 1-4. 2011 leguminosa aporta também fibra, ácido fólico, tiamina, potássio, magnésio e zinco, sento estes compostos de importância para a prevenção e tratamento de doenças cardiovasculares, diabetes, obesidade, constipação e câncer de cólon.

No Equador, esta leguminosa é consumida quase em todo o território nacional, sendo também fonte de renda para pequenos e médios produtores. 


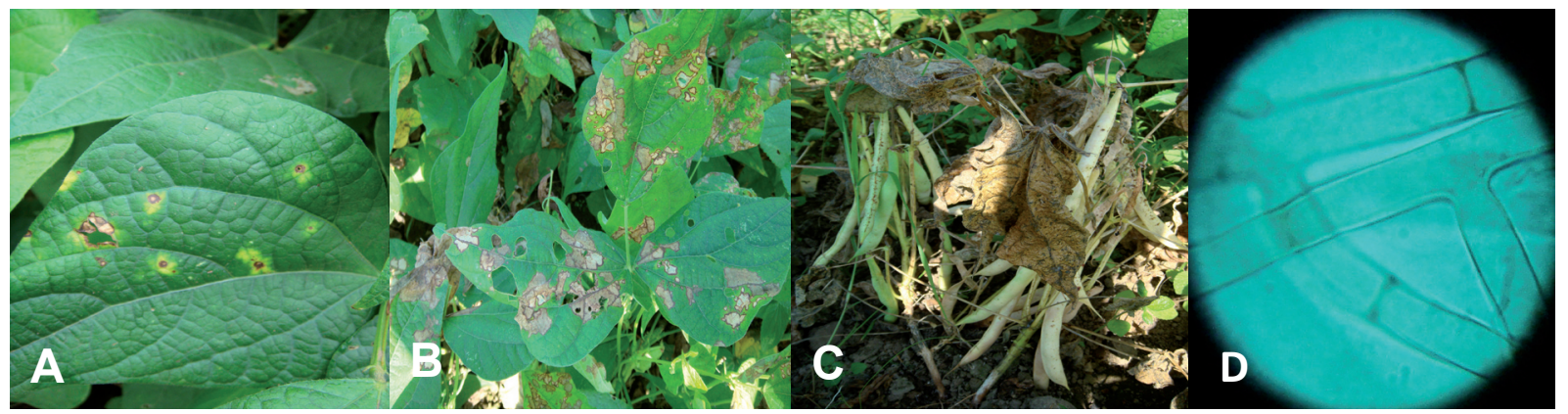

Figura 1. Sintomas: inicial (A), avançado (B) e perda quase total das folhas (C); e micélio (D) de mela ou podridão radicular causada por Rhizoctonia solani em plantas de feijoeiro.

Como em qualquer cultura, ocorrem fatores negativos que fazem que os produtores tenham danos e perdas na atividade agrícola causados pelos fitopatógenos (fungos, bactérias, nematóides e vírus).

Uma das doenças mais importantes é a podridão radicular de rizoctonia ou também denominado mela, causada pelo fungo necrotrófico Rhizoctonia solani Khun (teleomorfo Thanatephorus cucumeris). Segundo Bianchini et al. (2005) esta podridão é comum na América Latina e em outras regiões do mundo, sendo uma doença economicamente importante, responsável pela redução da população de plantas e redução da produção. No Equador, especificamente na parte central do Rio Guayas, esta doença tem uma importância única, pois é a que predomina sobre outras moléstias, convertendo se assim em um problema para quem cultiva a leguminosa. Em feijoeiro, $R$. solani pode induzir diferentes sintomas, incluindo tombamento pré ou pós-emergente, podridão de raízes e colo e podridão de vagens (Figura 1.) (Tolêdo-Souza et al., 2009).

Segundo Reis et al. (2004), as podridões radiculares, em geral, são consideradas as doenças de controle mais difícil e, considerando-se os danos que causam, têm recebido pouca atenção por parte dos pesquisadores. Podem, por isso, serem consideradas doenças abandonadas pelos pesquisadores.

Por outro lado, existem poucos trabalhos quantificando os danos ocasionados pelas doenças, entre eles tem-se em arroz (Marchetti \& Bollich, 1991, Araújo et al. 2006), aveia (Reis et al., 2008; Nerbass et al., 2010), trigo (King, 1976; Reis et al., 2000; Casa et al., 2004; Bohatchuk et al., 2008), milho (Nutter \& Jenco, 1992) e um realizado em feijoeiro por Bergamin Filho et al. (1997).

Este trabalho teve como objetivo determinar a redução no rendimento de grãos causados pela infecção natural de mela, em diferentes cultivares de feijão, na safra agrícola 2010, no município de Quevedo, Los Rios, Equador.

\section{Material e Métodos}

O trabalho foi conduzido na área experimental da Unidade de Pesquisa, Científica e Tecnológica UICYT da Universidade Técnica Estatal de Quevedo - UTEQ, em Quevedo, localizado em latitude Sul $1^{\circ}$ 00' 35', longitude Este $79^{\circ} 30^{\prime} 08^{\prime}$ ' e altitude de $77 \mathrm{~m}$, avaliando-se cinco linhas de feijão: SER 03, SER 08, SER 31, EVG - 16 - 08 e EVG - 06 - 103, e duas variedades: CAL 96 e INIAP 473. O experimento foi estabelecido em 01/06/2010. O delineamento experimental foi o de blocos casualizados, com sete tratamentos e quatro repetições. O gradiente da intensidade da doença e do rendimento foi gerado devido à variabilidade genética entre os materiais.

Aos 108 dias depois de semeadura, foi avaliada a severidade subjetiva da doença nos estádio fenológico R7 (desenvolvimento de legume) segundo Gepts (1988) citado por Hall (1991). A metodologia usada foi a subjetiva, quantificando a percentagem (\%) de área foliar necrosada.parcela ${ }^{-1}$. A colheita foi realizada em 01/10/2010 aos 124 dias pós-semeadura, para quantificação do rendimento.

Os dados de rendimento foram submetidos à análise de variância, com comparação de médias pelo teste de Tukey $(\mathrm{p}=0.05)$ no programa SAS 9.0 (SAS, 2002). Para estimar os danos causados pela doença foi utilizado o modelo de ponto crítico (James \& Teng, 1979; Bergamin Filho \& Amorim, 1996), realizando a análise de regressão entre a severidade foliar da doença (S) (variável independente), e o rendimento de grãos (R) (variável dependente), no estádio fenológico R7 de crescimento da cultura, obtendo-se a equação da função de dano.

Nesta safra agrícola, as condições climáticas foram favoráveis para infecção natural do patógeno e também pelo cultivo do feijoeiro em monocultura, conseguindo o gradiente da doença e do rendimento de grãos. 


\section{Resultados}

As cultivares de feijão SER 03, SER 08, SER 31 e EVG - 16 - 08 foram estatisticamente superiores aos demais cultivares para a severidade da doença. Comportamento similar foi encontrado na variável rendimento, onde as linhas SER 03, SER 08 e SER 31 foram superiores estatisticamente aos demais tratamentos (Tabela 1).

Tabela 1. Características físicas do grão, severidade (\%) de Rhizoctonia solani e rendimento.hectare ${ }^{-1}$ das linhagens e variedades de feijão estudadas. Quevedo, Equador. Safra 2010

\begin{tabular}{|c|c|c|c|}
\hline $\begin{array}{l}\text { Linhagens de } \\
\text { feijoeiro }\end{array}$ & Características físicas do grão & $\begin{array}{c}\text { Severidade } \\
(\%)\end{array}$ & $\begin{array}{c}\text { Rendimento } \\
\left(\text { kg.ha }^{-1}\right)\end{array}$ \\
\hline SER 03 & Mediano. preto avermelhado & 38.0 & $3,720.00 \mathrm{a}$ \\
\hline SER 08 & Mediano. preto avermelhado & 38.0 & $3,350.00 \mathrm{a}$ \\
\hline SER 31 & Mediano. preto avermelhado brilhante & 25.5 & $3,112.50 \mathrm{a}$ \\
\hline CAL 96 & Grande. pardo avermelhado escuro com listras creme & $88.0 \mathrm{a}$ & $1,556.25$ \\
\hline $\mathrm{EVG}-16-08$ & Pequeno. circular e ovóide & 38.0 & $2,253.75 \quad b$ \\
\hline $\mathrm{EVG}-06-103$ & Grande. pardo avermelhado claro. arinhonado. cumprido & $81.8 \mathrm{ab}$ & $1,610.00 \quad b c$ \\
\hline INIAP 473 & Grande. marrom avermelhado. cumprido & $69.3 \mathrm{~b}$ & $1,648.75 \mathrm{bc}$ \\
\hline CV (\%) & & 13.69 & 11.71 \\
\hline
\end{tabular}

${ }^{1}$ Médias seguidas pela mesma letra na coluna não diferem estatisticamente pelo teste de Tukey a $5 \%$ de probabilidade.

A equação da função de dano obtida pela relação entre o rendimento de grãos e a severidade foliar da doença (Figura 2.), foi $\mathrm{R}=4,257.5-33.16$ $\mathrm{S}$. onde $\mathrm{R}$ é o rendimento e $\mathrm{S}$ a severidade (\%) da doença. com $R^{2}=0.82$ e $p<0.0001$. Com a equação resultante foi normalizada, sendo $R=1,000.0-7.79 \mathrm{~S}$. O coeficiente de determinação foi menor do que o de Araújo et al. (2006), trabalhando com o patossistema Oriza sativa- $R$. solani achando valores de 0.97 a 0.99 . Este se deve a que eles trabalharam nos danos ocorridos

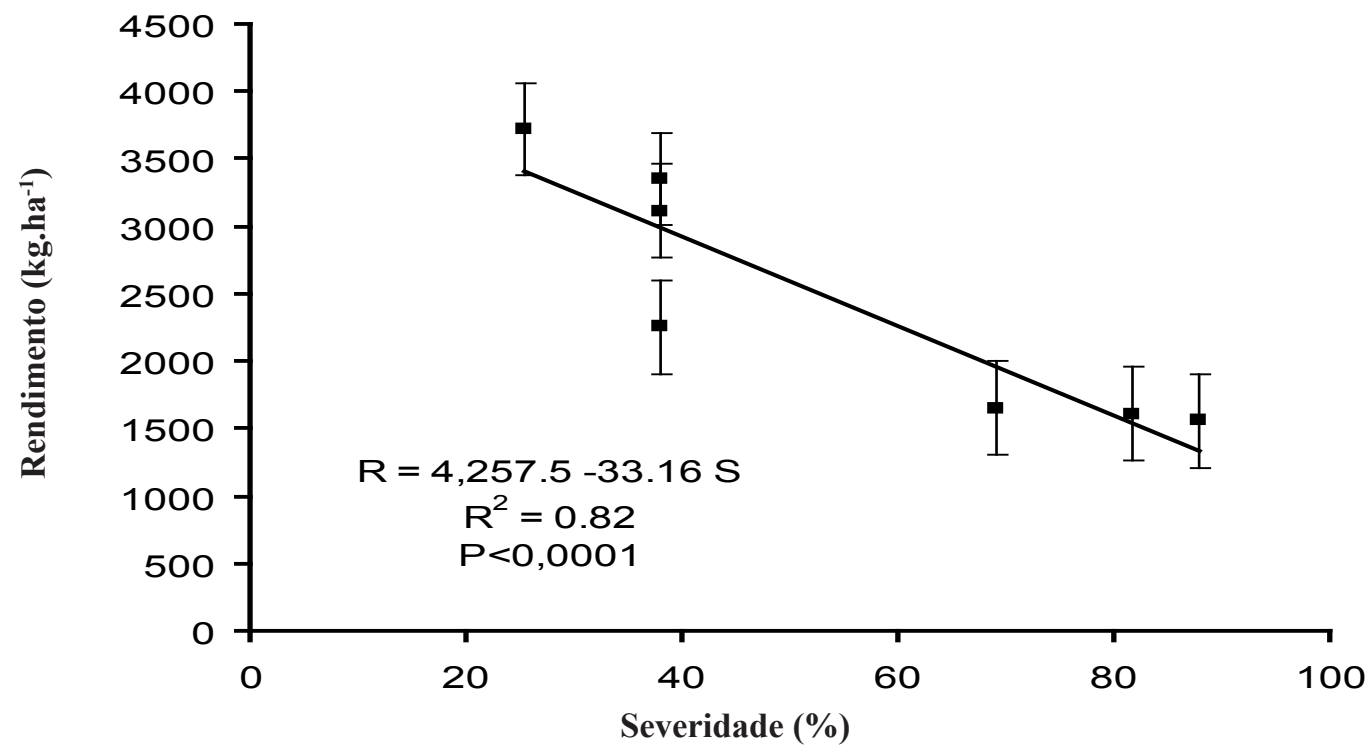

Figura 2. Relação entre rendimento de grãos e severidade (\%) de Rhizoctonia solani geradas pela reação de linhagens e variedades de feijoeiro. Quevedo. Equador. Safra 2010. 
Garcés.

nos componentes de produtividade (comprimento da panícula. porcentagem de espiguetas vazias e massa de grãos panícula ${ }^{-1}$ ) do arroz.

\section{Conclusão}

Os resultados da presente investigação mostraram que o dano no estádio fenológico R7 pode ser quantificado com o método de ponto crítico.Concluiuse que a equação gerada da função de dano pode ser utilizada no cálculo do limiar de dano econômico (LDE), sendo esta uma alternativa indicadora do momento para a aplicação de controle químico da mela em cultivares suscetíveis. mantendo a rentabilidade do agricultor e ser amigável com o meio ambiente.

Recomenda-se a necessidade da continuidade deste tipo de pesquisas (quantificação de danos) em não só em feijoeiro. se no em outras culturas, locais e anos em cultivares com reações semelhantes ou diferentes à doença avaliada neste trabalho. ou com outras doenças.

\section{LiTERATURA CITADA}

Araújo. L. G.. A. S. Prabhu. e G. B. da.. Silva. 2006. Método de perfilhos únicos para estimar os danos causados pela queima-da-bainha nos componentes de produtividade do arroz. Fitopatologia Brasileira. 31:199-202.

Araya. F. C. M. y F. J. C. Hernández. 2006. Guía para la Identificación de Enfermedades del Fríjol más Comunes en Costa Rica. MAG. San José.

Bergamin Filho. A. e L. Amorim. 1996. Doenças de Plantas Tropicais: epidemiologia e controle econômico. Agronômica Ceres. São Paulo.

Bergamin Filho. A.. S. M. T. P. G. Carneiro. C. V. Godoy. L. Amorim. R. D. Berger. and B. Hau. 1997. Angular leaf spot of Phaseolus beans: relationships between disease. healthy leaf area. and yield. Phytopathology. 87:506-515.

Bianchini. A.. A. C. Maringoni e S. M. T. P. G. Carneiro. 2005. Doenças do feijoeiro (Phaseolus vulgaris). In: Kimati. H.. L. Amorim. J. A. M. Rezende. A. Bergamin Filho e L. E. A. Camargo. Vol. 2. (4 Ed.). Manual de Fitopatologia. Agronômica Ceres. São Paulo.

Bohatchuk. D. A.. R. T. Casa. A. Bogo. J. P. R. Kuhnem. E. M. Reis. e E. N. Moreira. 2008. Modelo de ponto crítico para estimar danos de doenças foliares do trigo em patossistema múltiplo. Tropical Plant Pathology. 33: 354-360.
Casa. R. T.. E. M. Reis. M. M. C. Blum. A. Bogo. O. Scheer. e T. Zanata. 2004. Danos causados pela infecção de Gibberella zeae em trigo. Fitopatologia Brasileir.a 29:289-293.

Hall, R. 1991. Compendium of bean diseases. APS Press. Minnesota.

James. W. C. and P. S. Teng. 1979. The quantification of production associated with plant disease. Applied Biology. 4:21-267.

King. J. E. 1976. Relationship between yield loss and severity of yellow rust recorded on large number of single stems of wheat. Plant Pathology. 25:172177.

Marchetti. M. A and C. N. Bollich. 1991. Quantification of the relationship between sheath blight severity and yield loss in rice. Plant Disease. 75:773-775.

Nerbass. J. J. M.. R. T. Casa. J. P. R. Kuhnem e F. A. G. Bogo. 2010. Modelos de pontos críticos para relacionar o rendimento de grãos de aveia branca com a intensidade de doença no patossistema múltiplo ferrugem da folha - helmintosporiose. Ciência Rural. 40:1-6.

Nutter. F. W. and J. H. Jenco. 1992. Development of a critical-point yield loss model to estimate yield losses in corn caused by Cercospora zeae-maydis. Phytopathology. 82:990-994.

Reis. E. M.. R. Bezerra. O. Scheer. D. M. N. L. Martins. e C. Cardoso. 2004. Manejo das podridões radiculares em soja. Em: Reis. E. M. (Ed.). Doenças na Cultura da Soja. Série Técnica. Aldeia Norte. Passo Fundo.

Reis. E. M.. R. T. Casa e L. C. Bevilaqua. 2008. Modelo de ponto crítico para estimar danos causados pela ferrugem da folha da aveia branca. Summa Phytopathologica. 34(3):238-241.

Reis. E. M.. R. T. Casa. L. L.Hoffmann and C. M. Mendes. 2000. Effect of leaf rust on wheat grain yield. Fitopatologia Brasileira. 25(1):67-71.

SAS/STAT® Versão 9.0 do sistema SAS para Windows. Copyright 2002 SAS Institute Inc.. Cary. NC. USA.

Tolêdo-Souza. E. D.. M. L. Júnior. D. M. P. Silveira. e A. C. Café Filho. 2009. Interações entre Fusarium solani f. sp. phaseoli e Rhizoctonia solani na severidade da podridão radicular do feijoeiro. Pesquisa Agropecuária Tropical. 39(1):13-17. 